\title{
Flight Dynamic Response Analysis of the Flexible Aircraft Flying Through the Wind Field over Complex Terrains \\ Chu TANG ${ }^{1}$, Li-Bo WANG ${ }^{1}$
}

1The First Aircraft Institute of AVIC, Xi'an, Shaanxi, China 710089

Keywords: Complex terrain, Flight dynamics simulation, Flexible aircraft, Wind field.

\begin{abstract}
A numerical simulation method for the flight dynamic response of the elastic aircraft flying through the wind field over complex terrain is developed in this paper. The complex terrain model is established by the spline method based on the contour line of the map. The wind field is then simulated by the vortex lattice method in potential flow theory. Furthermore, the effects on the aerodynamic characteristics of the aircraft that induced by the structural flexibility and the wind velocity gradients are also considered. A numerical example is studied, and the result indicates that the structural flexibility would have a great influence on the dynamic response, when the aircraft flying through a complex wind field.
\end{abstract}

\section{Introduction}

During the flight, the aircraft is always affected by different kinds of atmospheric disturbances. The effects of the complex wind field on the flight control system, the structural loads, the dynamic responses, as well as the target tracking accuracy should be considered, when the aircraft is crossing a wind fields or executing a low-altitude mission. In order to analyse the dynamic response of the aircraft flying through a wind field, the equations of motion of the aircraft should combine with the wind field model. If necessarily, the coupling effects of the structural flexibility and the unsteady wind are also needed.

\section{Analysis Model for the Wind Field over Complex Terrains}

As shown in Figure 1, the complex terrain is represented by a surface, which is established by the surface spline method [1,2] basing on the contour line of the map. Then it is divided into panels containing vortex ring singularities. The singularity distribution is solved by combining with the potential flow theory and the boundary condition of terrain surface [3]. Finally, the wind field is simulated by linear superposition of the uniform stream and the induced velocity of vortex rings. Figure 2 shows the procedure for the wind field simulating over complex terrains.

Moreover, convective weather or severe convective weather often appears in the areas with complex terrains, because of the effects of the geography, the environment and other multiple factors. Therefore, a downburst model that represented by a vortex ring [4-6] is also adopted as shown in Figure 3.

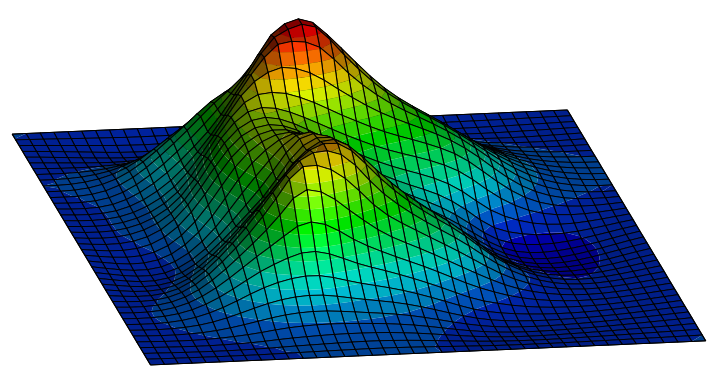

Figure 1. Result of the complex terrain simulation. 


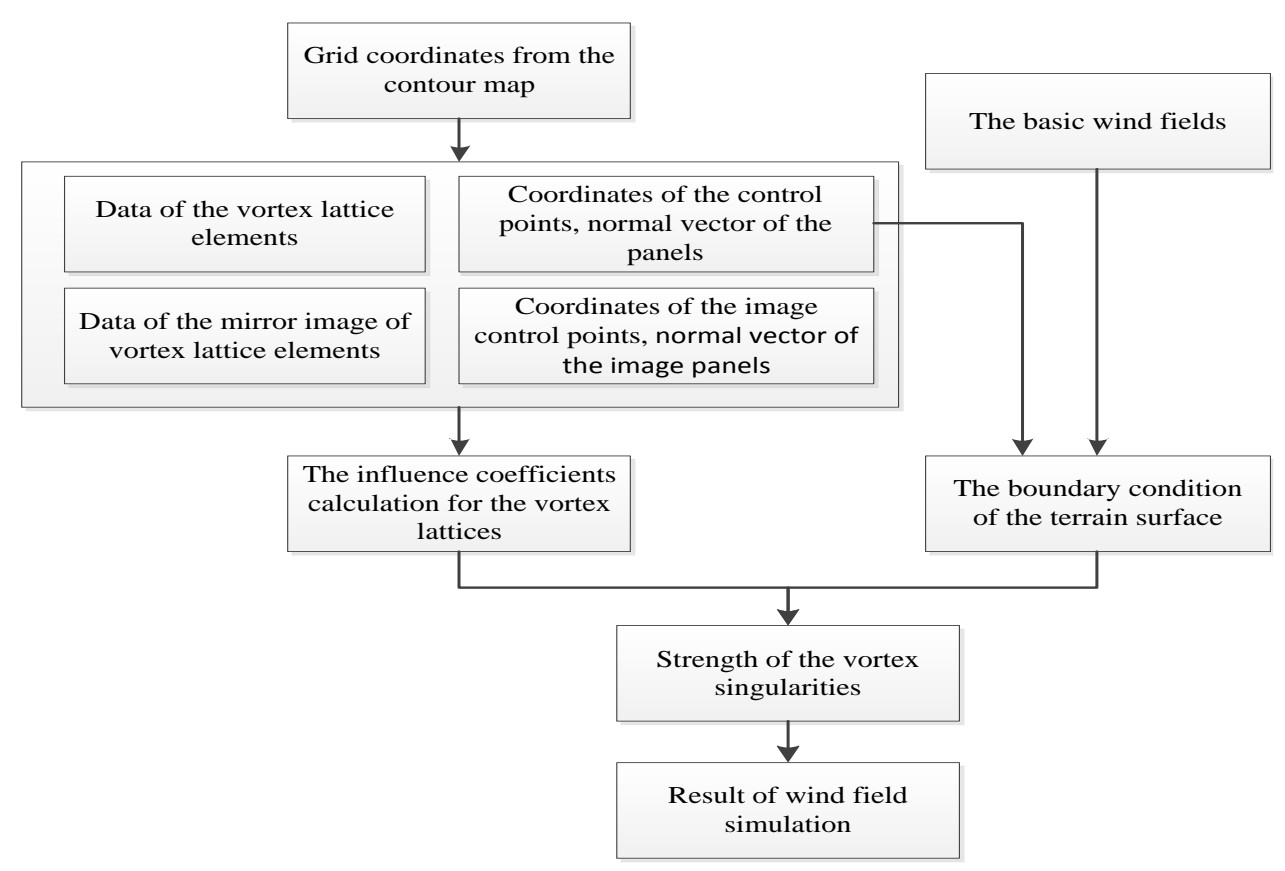

Figure 2. The flowchart for wind field simulating over complex terrains.

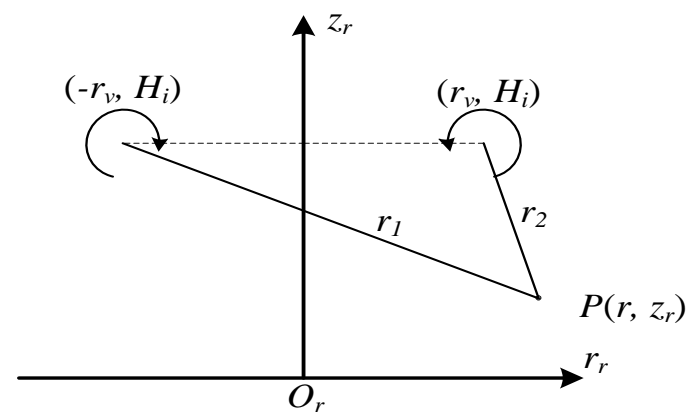

Figure 3. Vortex ring model for downburst simulation

\section{Theory for Flight Dynamics Simulation}

When establishing the analysis model for the flight dynamics of the aircraft, several kinds of reference frames have been used in this paper, in order to consider the effects of the rigid motions of the aircraft, the wind field and the structural flexibility. They are the earth-fixed reference frame $\left(S_{G}\right)$ $O_{g} X_{g} Y_{g} Z_{g}$, the body-fixed reference frame $\left(S_{B}\right) O_{x_{b} y_{b} z b}$, the atmosphere-fixed reference frame $\left(S_{A}\right)$ $O x_{a} y_{a} z_{a}$ and the air-trajectory reference frame $\left(S_{K}\right) O x_{k} y_{k} z_{k}$, respectively. The rotation of the earth relative to the inertial axes is neglected, and the earth axes $S_{G}$ is used as an inertial frame. The orientation of the other three reference frames relative to the earth axes can be given by seven angles, which are the angle of attack $\alpha$, the angle of sideslip $\beta$, the bank angle $\phi$, the elevation angle $\theta$, the azimuth angle $\psi$, the heading angle $\chi$ and the angle of climb $\gamma$ ([7]).

The motions of the aircraft are then represented by the translation and the rotation of the moving coordinate axes relative to the inertial reference frame. The location of the mass center of the aircraft in the earth axes is represented by the vector $\boldsymbol{R}_{G}=\left[\begin{array}{lll}X & Y & Z\end{array}\right]^{\mathrm{T}}$. The ground speed and the airspeed of the aircraft are represented by $\boldsymbol{V}_{B}=\left[\begin{array}{lll}u & v & w\end{array}\right]^{\mathrm{T}}$ and $\boldsymbol{U}_{B}=\left[\begin{array}{lll}u a & v_{a} & w_{a}\end{array}\right]^{\mathrm{T}}$ in the body axes, the wind velocity is written as $\boldsymbol{W}_{G}=\left[\begin{array}{llll}w_{x} & w_{y} & w^{z}\end{array}\right]^{\mathrm{T}}$ in the earth axes. The angular velocity of the body axes relative to the earth axes is written as $\omega_{B}=[p q r]^{\mathrm{T}}$ in the body axes.

\section{Equations of Motion of the Aircraft Flying Through a Wind Field}

The force equations of motion are 


$$
\begin{aligned}
\frac{d u}{d t}= & (r v-q w)-g \sin \theta+\frac{T}{m} \cos \varphi \\
& +\frac{1}{m}(-D \cos \alpha \cos \beta+L \sin \alpha) \\
\frac{d v}{d t}= & (p w-r u)+g \sin \phi \cos \theta \\
& +\frac{1}{m}(-D \sin \beta+C \cos \beta) \\
\frac{d w}{d t}= & (q u-p v)+g \cos \phi \cos \theta-\frac{T}{m} \sin \varphi \\
& -\frac{1}{m}(D \sin \alpha \cos \beta+C \sin \alpha \sin \beta+L \cos \alpha)
\end{aligned}
$$

The moment equations of motion are

$$
\begin{aligned}
& I_{x} \dot{p}-I_{x z} \dot{r}+\left(I_{z}-I_{y}\right) q r-I_{x z} p q=L_{A} \\
& I_{y} \dot{q}+\left(I_{x}-I_{z}\right) p r+I_{x z}\left(p^{2}-r^{2}\right)=M_{A}+M_{T} \\
& I_{z} \dot{r}-I_{x z} \dot{p}+\left(I_{y}-I_{x}\right) p q+I_{x z} q r=N_{A}
\end{aligned}
$$

According to the classic flight dynamics theory, the aerodynamic characteristics of the aircraft is represented by the aerodynamic coefficients and derivatives, likes

$$
\begin{aligned}
C_{D}= & C_{D 0}+A C_{L}^{2} \\
C_{S}= & C_{S \beta} \beta+C_{S \delta_{r}} \delta_{r}+C_{S \delta_{a}} \delta_{a}+C_{S p} \frac{c}{2 U} p+C_{S r} \frac{c}{2 U} r \\
C_{L}= & C_{L \alpha}\left(\alpha-\alpha_{0}\right)+C_{L \delta_{e}} \delta_{e}+C_{L q} \frac{c}{2 U} q+C_{L \dot{\alpha}} \frac{c}{2 U} \dot{\alpha} \\
C_{l}= & C_{l \beta} \beta+C_{l \delta_{r}} \delta_{r}+C_{l \delta_{\alpha}} \delta_{\alpha}+C_{l \dot{\beta}} \frac{b}{2 U} \dot{\beta}+C_{l p} \frac{b}{2 U} p \\
& +C_{l r} \frac{b}{2 U} r \\
C_{m}= & C_{m 0}+C_{m \alpha} \alpha+C_{m \delta_{e}} \delta_{e}+C_{m q} \frac{c}{2 U} q+C_{m \dot{\alpha}} \frac{c}{2 U} \dot{\alpha} \\
C_{n}= & C_{n \beta}+C_{n \delta_{r}} \delta_{r}+C_{n \delta_{\alpha}} \delta_{\alpha}+C_{n \dot{\beta}} \frac{b}{2 U} \dot{\beta}+C_{n p} \frac{b}{2 U} p \\
& +C_{n r} \frac{b}{2 U} r
\end{aligned}
$$

The kinematic equations of the translation of the aircraft are

$$
\begin{aligned}
\dot{X}= & u \cos \theta \cos \psi+v(\sin \phi \sin \theta \cos \psi-\cos \phi \sin \psi) \\
& +w(\cos \phi \sin \theta \cos \psi+\sin \phi \sin \psi) \\
\dot{Y}= & u \cos \theta \cos \psi+v(\sin \phi \sin \theta \sin \psi+\cos \phi \cos \psi) \\
& +w(\cos \phi \sin \theta \sin \psi-\sin \phi \sin \psi) \\
\dot{Z}= & -u \sin \theta+v \sin \phi \cos \theta+w \cos \phi \cos \theta
\end{aligned}
$$

The kinematic equations of the rotation of the aircraft are

$$
\left\{\begin{array}{c}
\dot{\phi}=p+(q \sin \phi+r \cos \phi) \tan \theta \\
\dot{\theta}=q \cos \phi-r \sin \phi \\
\dot{\psi}=(q \sin \phi+r \cos \phi) / \cos \theta
\end{array}\right.
$$




\section{Effects of The Wind Field on the Motion of the Aircraft}

The relationship of the airspeed, the ground speed and the wind speed is

$$
\mathbf{U}_{B}=\mathbf{V}_{B}-\mathbf{L}_{B G} \mathbf{W}_{G}
$$

where, $\boldsymbol{L}_{B G}$ is the coordinate transformation matrix between the earth axes and the body axes. According to the definition of the angle of attack and the angle of sideslip, there are

$$
\left\{\begin{array}{l}
\tan \alpha=\frac{w_{a}}{u_{a}} \\
\sin \beta=\frac{v_{a}}{U}
\end{array}\right.
$$

where, $U$ is the airspeed of the aircraft, $U=\left(u_{a}^{2}+v_{a}^{2}+w_{a}^{2}\right)^{1 / 2}$.

Derivatives of the angle of attack and the sideslip angle with respect to time are

$$
\left\{\begin{array}{c}
\dot{\alpha}=\frac{u_{a} \dot{w}_{a}-\dot{u}_{a} w_{a}}{u_{a}^{2}+w_{a}^{2}} \\
\dot{\beta}=\frac{\dot{v}_{a}\left(u_{a}^{2}+w_{a}^{2}\right)-v_{a}\left(u_{a} \dot{u}_{a}+w_{a} \dot{w}_{a}\right)}{U^{2} \sqrt{u_{a}^{2}+w_{a}^{2}}}
\end{array}\right.
$$

where, $\dot{u}_{a}, \dot{v}_{a}$ and $\dot{w}_{a}$ are the derivatives with respect to the time of the airspeed velocity components in body axes. It could be obtained by equation (8), as

$$
\dot{\mathbf{U}}_{B}=\dot{\mathbf{V}}_{B}-\mathbf{L}_{B G} \dot{\mathbf{W}}_{G}-\dot{\mathbf{L}}_{B G} \mathbf{W}_{G}
$$

where, $\dot{\boldsymbol{L}}_{B G}$ is the derivative of the transformation matrix with respect to the time, $\dot{\boldsymbol{L}}_{B G}=-\tilde{\boldsymbol{\omega}}_{B} \boldsymbol{L}_{B G}$. According to the assumption of the frozen field assumption, the time derivative of the wind speed vector $\boldsymbol{W}_{G}$ is

$$
\dot{\boldsymbol{W}}_{G}=\boldsymbol{W}_{G}^{\prime} \dot{\boldsymbol{R}}_{G}
$$

where, $\boldsymbol{W}^{\prime}{ }_{G}$ is the gradient matrix of the wind speed.

Substitute all the results above to equation (8) yields

$$
\dot{\boldsymbol{U}}_{B}=\dot{\boldsymbol{V}}_{B}+\tilde{\boldsymbol{\omega}}_{B} \boldsymbol{L}_{B G} \boldsymbol{W}_{G}-\boldsymbol{L}_{B G} \boldsymbol{W}_{G}^{\prime} \boldsymbol{L}_{G B} \boldsymbol{V}_{B}
$$

\section{Corrections of the Aerodynamics}

The aerodynamic coefficients are corrected by considering the effects of the structural flexibility. The angular velocity of the aircraft with respect to the airstream is also corrected by considering the wind velocity gradients.

\section{Effects of the Structural flexibility}

The aerodynamic derivatives of a flexible aircraft could be written as

$$
\boldsymbol{C}_{F a}=\frac{1}{q S} \boldsymbol{D} \boldsymbol{\Phi}_{r}^{T} \frac{\partial \boldsymbol{f}_{A}}{\partial \boldsymbol{a}}+\frac{1}{S} \boldsymbol{D} \boldsymbol{Q}_{r e}\left(\boldsymbol{K}_{e e}-q \boldsymbol{Q}_{e e}\right)^{-1} \boldsymbol{\Phi}_{e}^{T} \frac{\partial \boldsymbol{f}_{A}}{\partial \boldsymbol{a}}
$$

where, $q$ is the dynamic pressure; $S$ is the wing area; $D$ is a constant coefficient matrix; $\Phi_{\text {rand }} \Phi_{\varepsilon}$ are the mode matrix of the rigid motion and elastic vibration; $f_{\mathrm{A}}$ is the aerodynamic loads on the grids of the structural finite element model; $\boldsymbol{Q}_{\text {re }}$ and $\boldsymbol{Q}_{\text {ee }}$ are the aerodynamic influence coefficients matrix; $\boldsymbol{K}_{\mathrm{ee}}$ is the generalized stiffness matrix of the structure; $\boldsymbol{a}$ is the vector of trim variables of the aircraft, including the angle of attack $\alpha$, the angle of $\operatorname{sideslip} \beta$, et al. 


\section{Effects of Velocity Gradients in the Wind Field}

The equivalent angular velocity of the aircraft that induced by the wind velocity gradients could be represented as

$$
\begin{aligned}
& p_{G}=\left(\partial w_{z} / \partial y\right)_{B} \\
& q_{G}=-\left(\partial w_{z} / \partial x\right)_{B} \\
& r_{1 G}=-\left(\partial w_{x} / \partial y\right)_{B} \\
& r_{2 G}=-\left(\partial w_{y} / \partial y\right)_{B}
\end{aligned}
$$

Therefore, the roll rate, the pitch rate and the yaw rate of the aircraft, when calculating the aerodynamics of the aircraft, could be corrected as

$$
\begin{aligned}
& p_{r e l}=p-p_{G} \\
& q_{r e l}=q-q_{G} \\
& r_{r e l}=r-r_{1,2 G}
\end{aligned}
$$

\section{Flight Dynamics Simulation of the Aircraft}

The equations of motions of the aircraft, including the dynamic equations and the kinematic equations are all adopted from the classic flight dynamics theory of rigid aircraft. Whereas, the aerodynamic derivatives and the angular velocity in the aerodynamics are substituted by the results of equation (11) and (13). The simulation process of the flexible aircraft flying through a wind field is shown in Figure 4. In this paper, only the longitudinal part is studied.

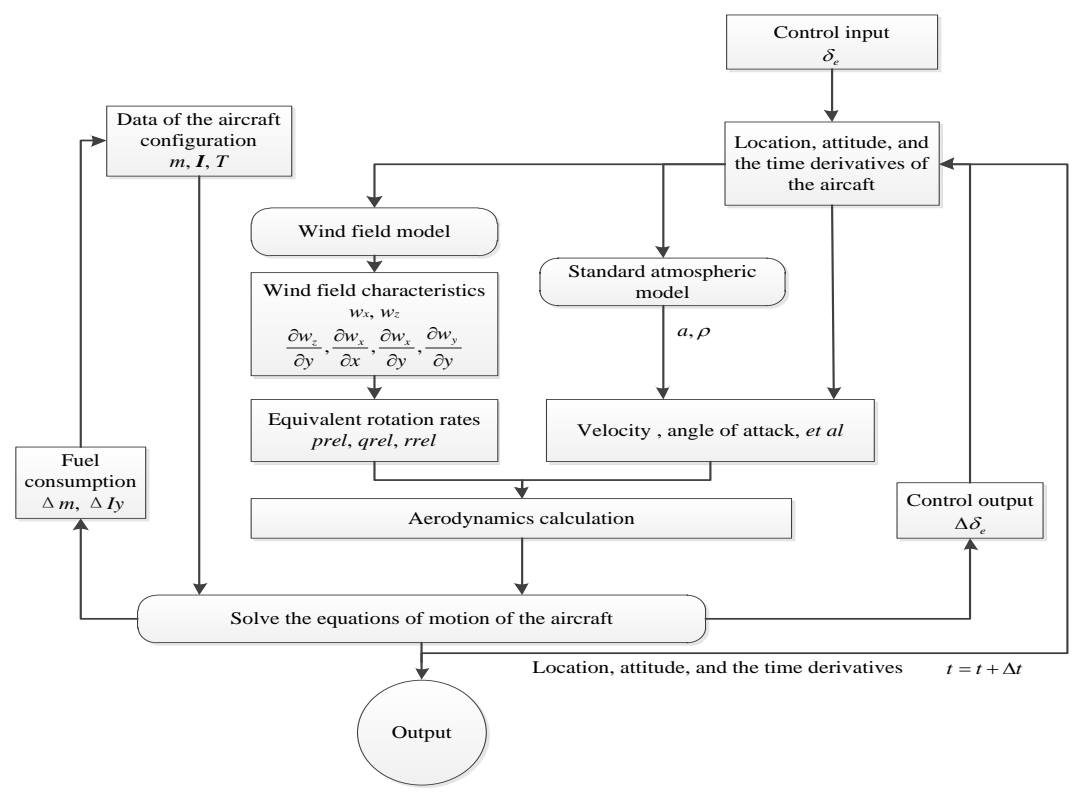

Figure 4. The flowchart for flight dynamics simulation of the aircraft.

\section{Numerical Example}

For instance, the longitudinal flight dynamic response of a flexible UAV with large-aspect-ratio wings is analyzed by using the method promoted in this paper.

The finite element model and aerodynamic model of the UAV are shown in Figure 5. The UAV has a conventional aerodynamic configuration, the wing span is $4.8 \mathrm{~m}$, and the gross takeoff weight is $20.0 \mathrm{~kg}$. Based on static aerodynamic analysis, the trim situation and the aerodynamic derivatives are calculated, when the true airspeed is $30 \mathrm{~m} / \mathrm{s}$. The result is shown in table 1 and table 2 . 
Figure 5. The structural model and aerodynamic model of the UAV

Table 1. The trim situation of the UAV

\begin{tabular}{|c|c|c|}
\hline Variables & Rigid aircraft & Flexible aircraft \\
\hline$C_{D}$ & 0.0338 & 0.0303 \\
\hline$C_{L}$ & 0.3682 & 0.3687 \\
\hline$C_{m}$ & 0.0 & 0.0 \\
\hline$\alpha$ & $3.7 \mathrm{deg}$ & $3.1 \mathrm{deg}$ \\
\hline$\delta_{e}$ & $1.3 \mathrm{deg}$ & $0.85 \mathrm{deg}$ \\
\hline$T$ & $17.92 \mathrm{~N}$ & $16.06 \mathrm{~N}$ \\
\hline
\end{tabular}

Table 2. The aerodynamic derivatives of the UAV

\begin{tabular}{|c|c|c|}
\hline Variables & Rigid aircraft & Flexible aircraft \\
\hline$C_{D 0}$ & 0.0295 & 0.0251 \\
\hline$C_{m 0}$ & -0.0038 & -0.0005 \\
\hline$C_{D^{\alpha}}$ & 0.0608 & 0.0893 \\
\hline$C_{L^{\alpha}}$ & 5.8777 & 8.6731 \\
\hline$C_{m^{\alpha}}$ & -0.3244 & -0.2913 \\
\hline$C_{m \dot{\alpha}}$ & -2.7003 & -2.7114 \\
\hline$C_{L q}$ & 7.0003 & 7.8849 \\
\hline$C_{L q}$ & -9.0010 & -9.0381 \\
\hline$C_{D \delta e}$ & 0.0200 & 0.0200 \\
\hline$C_{L \delta e}$ & -0.3305 & -0.3464 \\
\hline$C_{m \delta e}$ & 1.1133 & 1.1088 \\
\hline
\end{tabular}

By using the corrected aerodynamics, both of the dynamic responses of the rigid and flexible aircrafts have simulated. The wind field used for simulation is induced by complex terrains and a downburst, the distribution of the wind velocity is shown in Figure 6 . The initial location of the aircraft is $X_{0}=-2000 \mathrm{~m}, H_{0}=1500 \mathrm{~m}$. The flight paths of the rigid and flexible aircraft are illustrated in Figure 7 . The results show that, there is a big difference between the flight paths of the elastic aircraft with the rigid one.

For the flexible aircraft, the slope of lift curve is greater than the rigid one in the same airspeed. That leads to the variation of height and attitude is larger than the rigid aircraft, when the aircraft suffered of the vertical wind disturbance. 


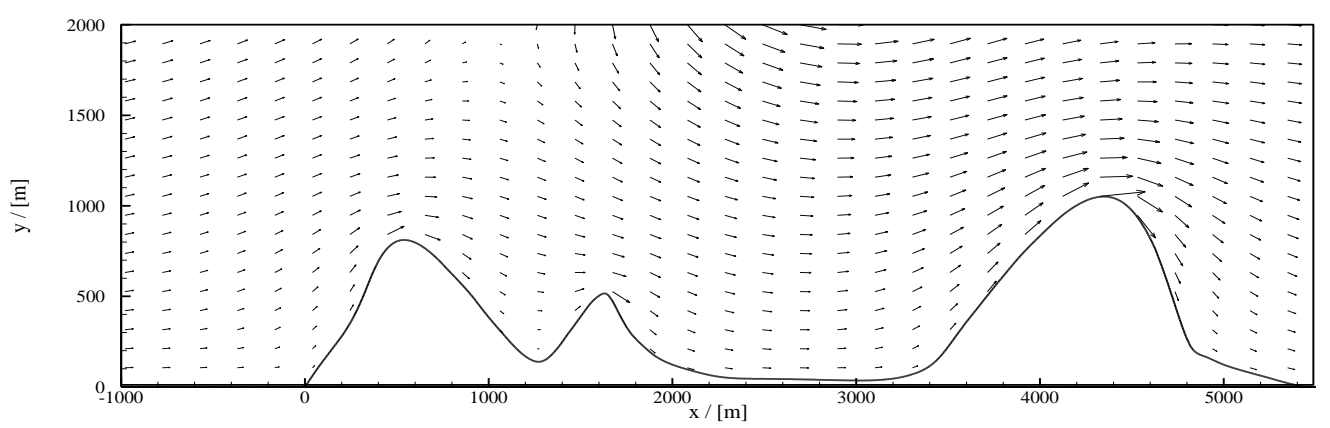

Figure 6. The wind field used for flight simulation

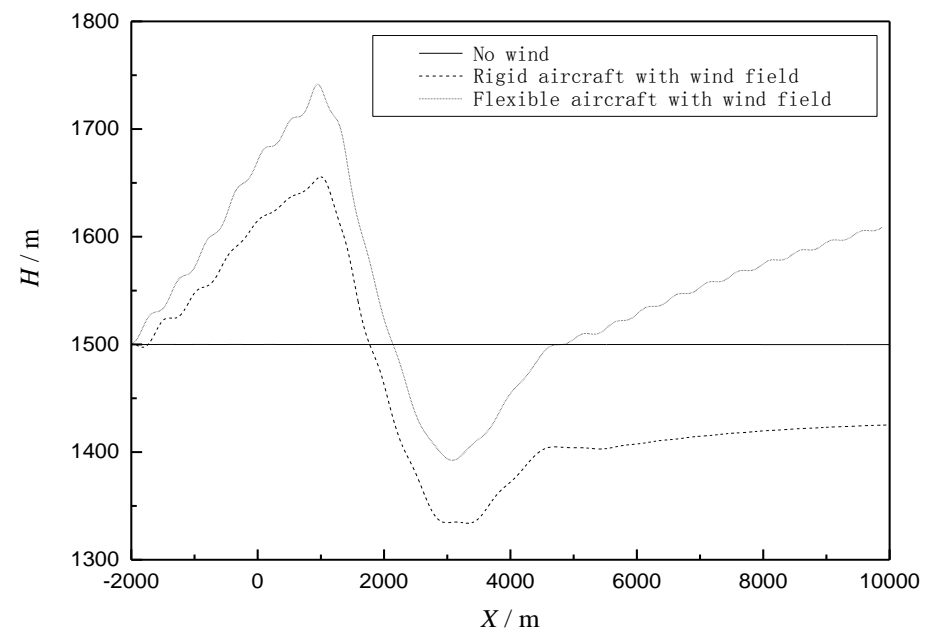

Figure 7. The flight paths of the rigid and flexible aircraft

The results of dynamic response are shown in Figure $8 \sim$ Figure 11. Both results of the aircraft in rigid and elastic situations are illustrated. For the flexible aircraft, the change amplitude of the attitude is much more remarkably than the rigid situation. It also takes the elastic aircraft much longer time recovering to an initial situation than the rigid ones. It indicates that, the structural flexibility could have a great influence on the flight dynamic response of the aircraft, while flying through a complex wind field.

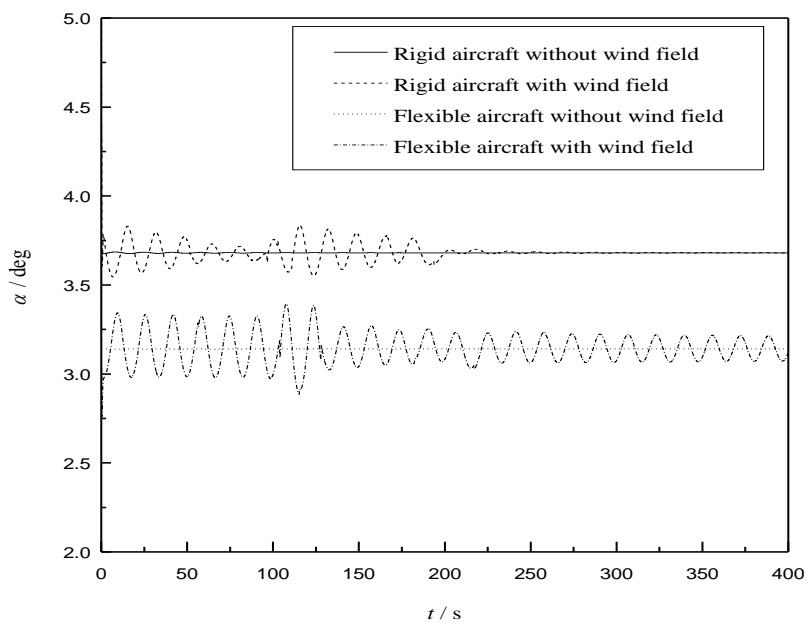

Figure 8 . The response of the angle of attack

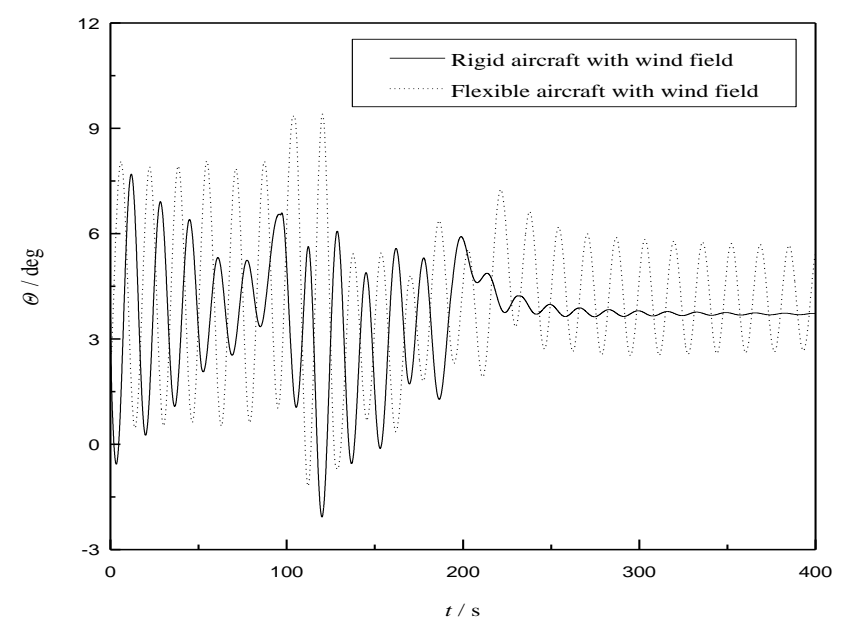

Figure 9. The response of the pitch angle 


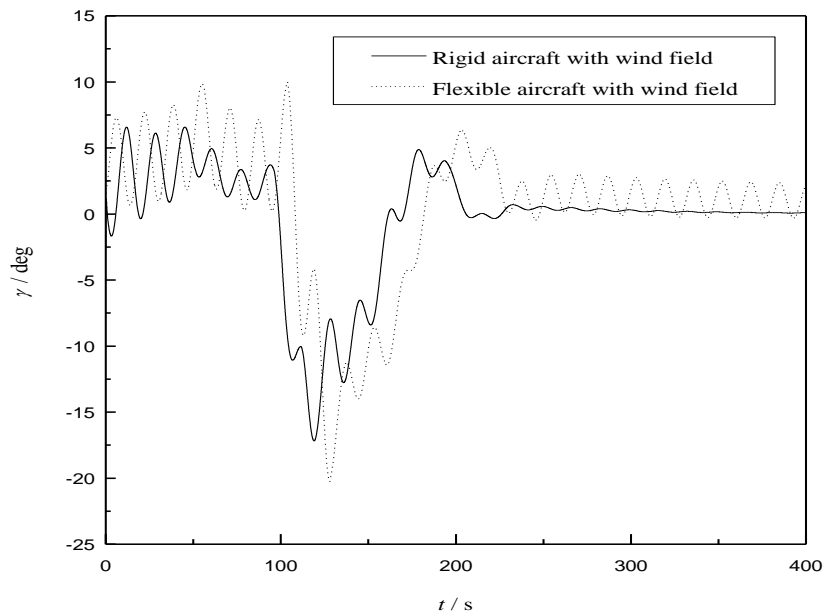

Figure 10. The response of the flight path climbing angle Figure 11. The response of the pitch rate

\section{Conclusion}

(1) The complex terrain is established by the spline method based on the contour line of the map. The wind field is then simulated by the vortex lattice method in potential flow theory.

(2) A numerical simulation method for the flight dynamic response of the elastic aircraft flying through the wind field over complex terrain is developed in this paper. The effects on the aerodynamic characteristics of the aircraft that induced by the structural flexibility and the wind velocity gradients are considered.

(3) A numerical example is studied, and the result indicates that the flexibility would have a great influence on the dynamic response, when the aircraft flying through a complex wind field.

\section{References}

1. Harder R L, Desmarais R N. Interpolation using surface splines. Journal of Aircraft. 1972, 9(2): $189-191$

2. Rodden W P, Mcgrew J A, Kalman T P. Comment on 'Interpolation Using Surface Splines. Journal of Aircraft. 1972, 9(12): 869-871

3. Tang Chu, Hong Guanxin. Simulation of wind field over complex terrain base on elevation data. Journal of Beijing University of Aeronautics and Astronautics. 2014, 40(3)

4. Woodfield A, Wood J F. Worldwide experience of wind shear during 1984 1982. AGARDCP, 1983

5. M. Ivan. A ring-vortex downburst model for flight simulator. Journal of Aircraft, 1986, 3(23): 232 236

6. Zhu S, Etkin B. Model of the wind field in a downburst. Journal of Aircraft, 1985, 22 (7): 595 601

7. Bernard E. Dynamics of atmospheric flight. Dover Publications. INC., 2005 